\title{
Estrategias Empresariales para una Población Vulnerable en Colombia
}

\author{
Ana M. Serrano(1)* Sandra C. Tiuzo ${ }^{(2)}$ y Martha S. Martínez ${ }^{(2)}$ \\ (1) Universidad Pedagógica y Tecnológica de Colombia, Facultad Seccional Duitama, Escuela de Administración de \\ Empresas Agropecuarias, Duitama, Colombia (e-mail: ana.serrano@uptc.edu.co) \\ (2) Universidad Pedagógica y Tecnológica de Colombia, Facultad Seccional Sogamoso, Escuela de Administración de \\ Empresas, Sogamoso, Colombia (e-mail: sandra.tiuzo@uptc.edu; martha.martinez@uptc.edu)
}

* Autor a quien debe ser dirigida la correspondencia

Recibido Mar. 5, 2019; Aceptado Abr. 22, 2019; Versión final Jun. 26, 2019, Publicado Dic. 2019

\section{Resumen}

Se presenta una revisión documental de estudios relacionados con estrategias empresariales para una población vulnerable que permita identificar cuáles han sido las estrategias de negocios que pueden implementarse en dicha población. Esta está ubicada en la estación de tren Siatame y el Durazno en el municipio de Sogamoso, en Colombia. El trabajo se desarrolló a través de una metodología cualitativa implementando un análisis documental. La información fue recopilada y subdividida en temas como estudios sobre población vulnerable, estrategias de negocios y asociatividad. La revisión destaca los estudios más representativos en los diferentes contextos y que fueron tomados como guía metodológica. Se concluye que deben ejecutarse estrategias empresariales enfocadas en la asociatividad, especialmente en ideas de emprendimientos para mejorar la calidad socioeconómica de la población.

Palabras clave: población vulnerable; estrategia empresarial; asociatividad; emprendimiento

\section{Business Strategies for a Vulnerable Population in Colombia}

\begin{abstract}
A documentary review of studies related to business strategies for a vulnerable population is presented with the objective of identifying which business strategies could be implemented in such a sector. This is located in the train station Siatame and El Durazno in the municipality of Sogamoso, in Colombia. The work was developed through a qualitative methodology implementing a documentary analysis. The information was collected and subdivided into topics such as studies on vulnerable population, business strategies, and associativity. The review highlights the most representative studies in the different contexts and that were taken as a methodological guide. It is concluded that entrepreneurial strategies focused on associativity, mainly on entrepreneurship ideas, must be implemented to improve the socioeconomic quality of the population.
\end{abstract}

Keywords: vulnerable population; business strategy; associativity; entrepreneurship 


\section{INTRODUCCIÓN}

La vulnerabilidad en Colombia se evidencia con mayor énfasis en las personas cuya naturaleza o debido a determinada circunstancia se encuentran expuestas a la exclusión, la pobreza, el efecto de inequidad y la violencia de todo orden (DNP, 2015). Dentro de los grupos vulnerables en Colombia se encuentran las personas con discapacidad, adultos mayores, niños, niñas y adolescentes, mujeres, comunidad indígena, personas con orientación sexual diversa, víctimas del conflicto armado y venezolanos desplazados. Este último grupo presenta cifras de $2.6 \%, 7.5 \%, 34.8 \%, 32 \%, 5.72 \%$, y $4.7 \%$, de acuerdo con el DANE (2016) (Departamento Administrativo Nacional de Estadística). Pachón (2007) define la vulnerabilidad como la sensibilidad y la inseguridad del bienestar de los individuos, hogares y comunidades en situaciones de cambio. Igualmente, Foschiatti (2008) manifiesta que la vulnerabilidad se genera por la inestabilidad económica, las condiciones de pobreza, la fragmentación social y la situación de indefensión de la población ante riegos. En Colombia para el año 2016, según estadísticas del DANE (2016), las poblaciones que presentan mayor crecimiento de su vulnerabilidad se concentran en las cabeceras municipales y principalmente son aquellas relacionadas con pobreza extrema con un $12.1 \%$, en las ciudades pobladas y zonas rurales se muestra un $37.6 \%$. Las ciudades que más presentan esta situación son Barranquilla (21\%), Montería (31\%), y Cartagena (26.2\%). Asimismo, al interior del país Bogotá, Bucaramanga, Manizales y Medellín mostraron índices de pobreza de $11.6 \%, 10.6 \%, 14.6 \%$ y $14.1 \%$, respectivamente.

Según estudio presentado por el DNP (2015) (Departamento Nacional de Planeación), el departamento de Boyacá presenta un $39.3 \%$ de pobreza y un $13.7 \%$ de pobreza extrema generada por el desempleo, el cual tiene una tasa de $8.9 \%$ y trabajo informal de $73.2 \%$. La población vulnerable del municipio de Sogamoso está compuesta específicamente de desplazados, personas en situación de discapacidad, población infantil, adolescente y joven, y mujeres cabeza de familia. Para el año 2017, se evidenció un $40.93 \%$ de inclusión de desplazados de algunos departamentos de Colombia como lo son Cundinamarca, Meta y Bogotá D.C. los cuales carecen de recursos económicos, violencia intrafamiliar y de oportunidades laborales para lograr satisfacer las necesidades básicas. La población vulnerable especifica del municipio de Sogamoso, objeto de estudio, está constituida por adultos mayores, víctimas de desplazamiento y personas en situación de discapacidad con 57\%, 2.4\%, y 51.6\%, respectivamente, de acuerdo con la Secretaria local de salud (2016). Es importante destacar la falta de oportunidades y de generación de empleo dada por empresas regionales, es por esta razón la importancia de vincular tanto la academia, las empresas y el estado para ayudar a solucionar esta problemática tan marcada en el municipio del Sogamoso. Este artículo es resultado de la investigación de la Universidad Pedagógica y Tecnológica de Colombia, y su objetivo es llevar a cabo una revisión bibliográfica con el fin de analizar algunos estudios referentes a población vulnerable y estrategias empresariales.

\section{METODOLOGIA}

Se utilizó un enfoque cualitativo y se hizo un análisis documental a partir de textos recopilados de diversas bases de datos. Se consideraron estudios en los diversos contextos, y se hicieron búsquedas a partir de palabras clave como población vulnerable, estrategia empresarial y asociatividad. Posteriormente, se relacionaron estos estudios en una tabla de conocimiento, destacando las investigaciones que dieron un aporte significativo para la ejecución de la investigación. El proceso de investigación se dividió en tres fases:

Primera fase: recolección de la información. Con el fin de llevar a cabo la revisión documental, se establecieron los siguientes criterios de selección: a) Tipo de texto: artículos producto de investigación. b) Fuente: bases de datos como Scopus, Scielo, Web of Science, el DANE y el DNP. c) Temáticas: la asociatividad, la estrategia empresarial y la población vulnerable. d) Cronología: estudios realizados en los últimos 10 años. De los 500 artículos encontrados inicialmente, se utilizaron 120 que cumplieron con los criterios establecidos; este proceso duró aproximadamente cinco meses. Se encontraron investigaciones que definen el objeto de estudio, i,e., población vulnerable, como un proceso multidimensional relativo al riesgo o probabilidad de un individuo, hogar o comunidad de ser herido, lesionado o dañado ante cambios o permanencias de situaciones externas o internas. Adicionalmente, se hizo énfasis en los estudios realizados con estrategia empresarial, definida por calderón et al. (2010) como "un conjunto de acciones encaminadas a la consecución de una ventaja competitiva sostenible en el tiempo y defendible frente a la competencia, mediante la adecuación de los recursos y capacidades de la empresa y del entorno en el cual opera y que satisfaga los objetivos de los múltiples grupos participantes en ella" (p.18).

Segunda fase: análisis de la información. Este proceso se hizo mediante una matriz de conocimiento, en la cual se destacan aspectos como el objetivo de las investigaciones, metodología empleada, resultados y conceptos enfocados en población vulnerable, estrategia empresarial, asociatividad, y emprendimiento, entre otros. Se empleó una metodología con enfoque cualitativo. 
Tercera fase: escritura del artículo. A partir de la información recolectada y el análisis llevado a cabo, se dio paso al proceso de escritura del artículo basado en los parámetros para autores establecidos en la revista.

\section{RESULTADOS}

Como resultado se muestra el análisis de los diversos estudios fundamentales, tomando como foco diversos tipos de población como la infantil, mujeres cabeza de familia, personas en situación de extrema pobreza, personas con orientación sexual diversa, y jóvenes, entre otros, destacando que en el municipio de Sogamoso se encuentra este tipo de población vulnerable, dado que está localizado en un punto estratégico para que habitantes de otros departamentos como Casanare foco de conflicto armado en el pasado y actualmente el desplazamiento que viven los ciudadanos Venezolanos, los cuales han generado problemas de corte social y económico para este municipio. Con estos estudios los que se quiere es evaluar la relevancia y adaptarlos para el los habitantes que presentan este tipo de vulnerabilidad.

\section{Estudios Fundamentales}

Para los estudios sobre población vulnerable se han considerado diversas perspectivas para fortalecer los conceptos teóricos utilizados en los objetivos de la investigación, metodología y resultados. Del mismo modo se enfocan en especificaciones referentes al tema de estudio; destacando como población vulnerable en América Latina, la infantil, la familia de bajo recursos económicos y los desplazados.

En América Latina, se muestran estudios abordados por la ONU (Organización de Naciones Unidas) y la CEPAL (Comisión Económica para América Latina y el Caribe), en los cuales se identifica el incremento de población vulnerable debido a los impactos producidos por las formas de producción, las instituciones y los valores que caracterizan un nuevo patrón de desarrollo en los países. En esta investigación se hace un recorrido desde la década de los ochenta, hasta los noventa denominada "década muerta" debido a los diversos sucesos entre los cuales se destacan la caída del muro de Berlín, la disolución de la URSS, la crisis fiscal del estado y la insuficiencia de las políticas Keynesianas. En dichos eventos se involucra a América Latina a partir del capitalismo industrializado, originando debilidades económico-tecnológicas, el retiro de la actividad productiva y la pérdida de mercado, especialmente en los años noventa. Todo ello generó vulnerabilidad social y conllevó a fenómenos de pobreza y desplazamiento en la mayoría de países en Latino América (Pizarro, 2001).

Dentro de los estudios realizados sobre población vulnerable en América Latina se encuentra uno en Brasil acerca de la vulnerabilidad social en el campo de los desastres (Souza,2006). En dicha investigación se relaciona la vulnerabilidad en efectos de salud humana con la sociedad, la economía y la cultura, y se explica la vulnerabilidad como la reducción de la capacidad de algunas poblaciones para sobrevivir, resistir o recuperarse de las situaciones de peligro y eventos tales como los riesgos o las enfermedades profesionales. Souza (2006) Identifica inicialmente la afectación de población vulnerable debido al manejo de residuos sólidos generados por una empresa cementera en Brasil; esta problemática social involucra a actores sociales, población e instituciones. Este estudio propone un enfoque cualitativo y busca dar a conocer los riesgos laborales y ambientales en los países con alta vulnerabilidad social; se utilizaron entrevistas y se hizo un análisis de documentos. Como resultado se explican estrategias de integración de conocimiento, la vinculación de los grupos interesados y el empoderamiento de los grupos vulnerables promoviendo así la seguridad y la salud de esta población.

Con respecto al estudio en población infantil, se encontraron estudios presentados por la UNICEF (Fondo de las Naciones Unidas para la Infancia), especialmente sobre tratamiento de la pobreza y vulnerabilidad de los niños y niñas, con el fin de demostrar los mecanismos existentes y potenciales de protección social para la reducción de tales fenómenos en Guinea Ecuatorial. Niño et al. (2012) muestran que la pobreza es alta en dicho país (76.8\%) y que afecta a los niños directa e indirectamente, ya que su relación con ella depende de su hogar. El objetivo de esta investigación fue brindar estrategias empresariales y sociales que permitieran llegar a la reducción de la pobreza, la vulnerabilidad y el riesgo entre los infantes en Guinea Ecuatorial. Se aplicó un enfoque sistémico basado en evidencia de la protección social que beneficia a los niños y no a los programas e intervenciones dispersos que actualmente existen.

Rosenbergl (2008), afirma que en África más de "12 millones de niños menores han quedados huérfanos por el SIDA" (p.25), ocasionando que estos se conviertan en una población vulnerable dada su "privación económica, nutricional y psicosocial" (p. 6). Además, estos niños abandonan sus estudios y son discriminados socialmente. Este estudio refleja la importancia que las ONG están dando a este tema, brindando recursos económicos y prebendas para ayudar a la financiación y lograr la reducción y abandono de los huérfanos y niños vulnerables. Según Thurmana (2008), el compromiso de los países es indispensable para ayudar y apoyar a comunidades vulnerables como los huérfanos. En este estudio hecho en Ruanda después del 
genocidio de 1994, se destacaron los llamados "huérfanos dobles". Es primordial el apoyo hacia poblaciones como los huérfanos y jóvenes vulnerables para favorecerlos, pero se detecta que la comunidad de Ruanda pone la responsabilidad en manos de las autoridades competentes y de las ONG. Otra problemática es el abandono de los hijos en orfanatos por parte de sus padres quienes creen que en estos lugares están seguros y protegidos. Como resultado, se destacan las diversas estrategias para lograr el cuidado de esta población vulnerable, entre ellas, el apoyo de la comunidad hacia huérfanos y jóvenes vulnerables mediante la intervención de las entidades competente y las ONG con el fin de evitar que su número se incremente.

Engle (2011) realizó un estudio relacionado con el desarrollo cognitivo de la primera infancia en los paises de medianos y bajos ingresos en el cual se destaca la estrategia educativa de apoyo al sostenimiento escolar. Buvinic (1991), en el informe presentado al CEPAL en 1970, evidenció la necesidad de prestar atención a la vulnerabilidad de hogares cuyas madres desempeñaban un papel como cabezas de familia y, por lo tanto, tenían el control y mando; gracias a dicho informe, se crearon políticas en América latina para el control y apoyo sobre el tema.

En los años ochenta y noventa, teniendo en cuenta los cambios económicos presentados en América Latina, especialmente el caribe, se hicieron algunas modificaciones en las estrategias para el desarrollo de la mujer y sus hogares, y se generó un amplio conocimiento a este respecto. La investigación realizada en New York y el centro internacional de Washington sobre la estructura familiar 'mujer como cabeza de hogar' en países en vías de desarrollo dan como resultado la evaluación del conocimiento pertinente y la creación de políticas para el fortalecimiento de estos tipos de hogares que se han incrementado considerablemente. (Buvinic,1991).

Entre los estudios enfocados en el desplazamiento se resalta el presentado por Gómez (2007), en el cual se analiza el "desempleo y la tasa de desocupación" con el incremento de desplazados en la ciudad de Medellín. En esta investigación se mostró que durante 1995 se presentó un aumento de $4.77 \%$ de desplazamiento, originado especialmente por la violencia que se vivió en el departamento de Antioquia. Por otra parte, en Bogotá, Jaramillo (2006) llevó a cabo una investigación en las comunidades de Ciudad Bolívar, Suba, Puente Aranda y Bosa y destacó la problemática social y económica que estaba originando la comunidad desplazada. Este estudio contó con la participación de investigadores expertos que entrevistaron y aplicaron encuestas acerca del origen del desplazamiento y las problemáticas que este ocasionaba para la ciudad de Bogotá. Como resultado, se destacan la creación de alianzas estratégicas con sectores productivos para programas de generación de ingresos y empleo, y la conformación de comités locales de atención para la población desplazada.

La Universidad de Los Andes también reporta un estudio sobre el desplazamiento en Colombia debido al conflicto armado; en él se aplicaron encuestas a 2322 hogares desplazados con el fin de determinar los cambios sociales y económicos que ellos sufrieron a causa del desplazamiento. Este estudio demostró que los hogares desplazados se enfrentan a situaciones de extrema pobreza; puesto que no pueden generar ingresos, las familias se ven obligadas a migrar de sus territorios y, la mayoría de las veces, no pueden vender sus activos, lo que ocasiona desigualdad y bajo nivel de vida para la población. Además, las oportunidades laborales son remotas ya que sus fortalezas laborales están basadas en el sector agrícola, pero en la ciudad ellos no pueden hacer uso de dichas habilidades. Los desplazamientos también originan desintegración familiar, ya que los miembros de estas familias, en su mayoría, son asesinados o reclutados por los grupos al margen de la ley (Ibañez y Moya 2010).

El estudio de la Universidad Nacional titulado "Emprendimiento social: factores críticos de éxito y fracaso para el mejoramiento del programa de generación de ingresos de la presidencia de la república de Colombia, dirigido a población en situación de desplazamiento por la violencia en Caldas" se realizó con el objetivo de dar a conocer los resultados de la creación y consolidación de pequeñas unidades de negocio implementadas en 2007, con el fin de satisfacer necesidades económicas y sociales. En esta investigación también se evaluaron los factores críticos de éxito y se propusieron alternativas o estrategias para mejorar la ejecución del programa de la Agencia Presidencial para la Acción Social de la Presidencia de la República de Colombia (Rendón, 2011).

Con respecto a la población vulnerable de jóvenes, la fundación Carolina Cealci hizo un estudio para identificar los programas de formación para el empleo, educación y trabajo y la problemática de inserción laboral con equidad para los jóvenes desempleados en Colombia. Se tomaron como referencia las ciudades donde más se presenta desempleo juvenil, i.e., Bogotá, Medellín, Cali, Manizales y Barranquilla (Arcoiris, 2008). Esta investigación ofrece alternativas para la formulación y ejecución de políticas para el empleo de los jóvenes vulnerables que carecen de un empleo formal. Montoya (2009) define la estrategia empresarial como "la elección de una posición valiosa y única afianzada o enraizada en sistemas de actividades que son muy difíciles de evaluar" (p. 23-44). Este autor analiza la estrategia teniendo como referencia a Minztberg (en Moore, 2011) quien resalta la importancia para el desempeño y el cumplimiento de las metas, logrando con 
esto una superioridad del competidor. Adicionalmente, Manso (2003), especifica que para las estrategias se formulan después de "haber identificado, evaluado y seleccionado diferentes cursos de acción donde se debe tener en cuenta el establecimiento de objetivos, identificación de brechas, formulación de cursos de acción y grado de eficiencia".

Según Martínez y Milla (2005), para establecer estrategias empresariales debe tenerse como referencia el análisis del entorno con el fin de "detectar tendencias y acontecimientos del pasado, presente y futuro de la sociedad" ( $p$.4-40). En este estudio, que analizó población vulnerable localizada desde hace más de 10 años en el municipio de Sogamoso, fue indispensable revisar el entorno, las causa y generalidades que ocasionan la baja calidad de vida y pobreza para diseñar estrategias y soluciones alternativas que permitieran ayudar a fortalecer sus actividades económicas y ofrecer alternativas económicamente sostenibles y rentables por parte del municipio.

Para Amaya (2007), la formulación estratégica debe "convertirse en planes de acción concretos, con definición de responsables, para ello se debe proyectar el tiempo para cada uno de los proyectos estratégicos, definir los objetivos de las estrategias de cada área funcional dentro de los proyectos, así como diseñar planes de acción concretos". Asimismo, Aguilar (2010) resalta que la implementación de la estrategia "implica el patrón de decisiones a la consecución de un plan". Una vez establecidas estas estrategias, según Osorio et al. (2010), existe la necesidad de contar con un factor empresarial en las explicaciones teóricas del proceso de crecimiento económico y de ofrecer estrategias de emprendimiento que destaquen las ventajas.

Esta teoría del emprendimiento es considerada para Colombia como un estrategia empresarial fuerte para la generación de empleo, por tal razón se presentan diversas actividades desde el contexto académico con apoyo de entidades gubernamentales como el fondo emprender del Sena para la capacitación a este tipo de población; con aras a la inserción en la vida laboral y así mismo mejorar las condiciones de vida.

Para llegar al desarrollo e implementación de diversas estrategias, debe hacerse un análisis estratégico y establecer el objetivo, es decir, identificar las estrategias externas e internas que analicen las amenazas y oportunidades o fortalezas y debilidades en el ámbito externo. Para tal ámbito, Osorio et al. (2010) destacan el factor del entorno como una característica esencial para la formulación estratégica, en otras palabras, es importante que las organizaciones identifiquen los componentes económicos, tecnológicos y sociales.

Dentro de los estudios actuales se encuentra el presentado en Perú por Porto y Fernandes (2018) en el cual se diseñan estrategias empresariales especialmente de producción de alimentos ambientales sostenibles, en la avicultura, con el ánimo de mejorar la seguridad alimentaria de la población vulnerable de bajos recursos económicos vinculando el gobierno, la empresa y la sociedad. Esta investigación es importante destacarla puesto que evidencia la importancia de vincular los actores anteriormente mencionados para la inclusión de este tipo de población con el fin de mejorar las condiciones económicas de este tipo de población.

Se toman estos estudios partiendo de la necesidad de mostrar la importancia de la estrategia empresarial como eje fundamental para el acercamiento a este tipo de comunidad, partiendo del análisis del entorno, la responsabilidad que debe asumir el estado para lograr un acercamiento y generación de elementos fundamentales para el crecimiento económico de esta comunidad, a partir de la implementación de proyectos productivos que generen para que población pueda tener un sustento diario.

\section{Estrategia Empresarial de Asociatividad}

La Asociatividad, según Cervilla (2007) es un "mecanismo de cooperación entre empresas pequeñas y medianas donde cada empresa decide participar voluntariamente con los otros participantes para la búsqueda de un objetivo común" (p. 230). Ante la situación económica que está viviendo la población vulnerable, es importante llegar a esta asociatividad empresarial, teniendo en cuenta que para Kingsbury y Hayter (2006) esta es "la cooperación directa e implícita a través de canales informales, las relaciones bilaterales y multinacionales directas con otras empresas como proveedores, compradores y el estado" (p. 596). Estos mismos autores establecen que la asociatividad es vista como un modelo que permite "la implementación de estrategias colectivas y de carácter voluntario con el fin de alcanzar niveles de competitividad". Por lo tanto, se analizaron algunos estudios referentes a la asociatividad, destacándose los siguientes:

El estudio de Ubilla e Irigoye (2007) hace referencia a la importancia de la asociación especialmente de los trabajadores cartoneros informales que se dedicaban individualmente al desarrollo de esta labor; se evidenció que el principal problema es la falta de asociatividad, lo cual origina obstáculos del desarrollo social e individual de una comunidad. Esta investigación fue de tipo cuantitativo y utilizó una muestra de 80 socios dedicados al reciclaje que respondieron una encuesta y una entrevista. Como resultado se muestra la capacidad que tuvo 
esta población de asociarse y conformar un gremio sindical, utilizando el capital humado y del estado para lograr beneficios económicos y validación ante la comunidad y las autoridades gubernamentales. Esta investigación demostró que la asociatividad es indispensable para mejorar la calidad de vida y el desarrollo humano.

Igualmente, Mariño (2012) en su estudio desarrollado en el alto del Ricaurte, se enfocó en la asociación en los productores de tomate, especialmente, y obtuvo como resultado el mostrar la importancia de conformar redes empresariales para la actividad agrícola mediante un proceso de planificación estratégica asociativa que involucre a los miembros de la asociación con miras a proponer soluciones y proyecciones en el futuro. En el estudio realizado por Szmulewicz y Gutiérrez (2012) sobre la evolución de las habilidad asociativas en redes del agroturismo del sur de Chile, se presentan como resultados las principales debilidades de las redes empresariales. En cuanto a la asociatividad, se evidenció la falta de comunicación, de compromiso, sentido de pertenencia, liderazgo en proyectos planteados a largo y corto plazo y técnicas organizacionales para la toma de decisiones.

Es importante resaltar el estudio planteado por Rodríguez (2007) "Emprendimiento y Asociatividad como Herramienta de desarrollo social para la educación media” en el cual presenta un modelo para el diagnóstico, caracterización y el desarrollo del emprendimiento y asociatividad. Este investigador tuvo como población a estudiantes de dos colegios de Bogotá, con el propósito de evaluar su potencial de emprendimiento y asociatividad mediante talleres y posteriores capacitaciones sobre idea de negocio, plan de negocio, asociatividad y emprendimiento. Dicho modelo se plantea con el propósito de mejorar la educación media, direccionar al estudiante en estos temas e impactar comunidades socioeconómicamente vulnerables.

Dentro de los estudios sobre estrategia empresarial de asociatividad se encuentra "La Integración de la Sociabilidad y Asociatividad en el Trabajo con Familias Marginales" llevado a cabo con familias de micro campamentos de habitantes de la calle. Como resultado se muestra que las personas que viven en estas condiciones son jóvenes con hijos y sin un trabajo estable, lo que origina pobreza y desigualdad. Esta investigación, de tipo exploratorio y de carácter cualitativo, destaca variables que originan conflictos de convivencia como falta de unión entre ellos, la desorganización y la falta de agrupamiento para el desarrollo de actividades. Se plantean estrategias como la organización de redes empresariales con el fin de brindar protección y sostenibilidad a las microempresas que pretenden desarrollarse.

Un estudio realizado en la Universidad Tecnológica de Pereira, hace referencia a la importancia de la asociación como estrategia fundamental para el desarrollo competitivo de los productores de piña, los cuales no cuentan con tecnología ni capital humano especializado para el desarrollo del producto. Esta investigación plantea un modelo de asocitivadad para establecer normas claras de funcionalidad que integren ordenadamente los pasos mínimos para dirigir adecuadamente una empresa o asociación" (Arias et al. 2010). Este modelo promovió la competitividad en el mercado, ya que se creó una empresa dedicada a la comercialización del producto para que las familias continuaran con su producción y ventas que actualmente posee; los excesos y la producción defectuosa se lleva a la nueva empresa creada con los productores de piña y esta, a su vez, se encarga de suministrarla al mercado nacional. La Estrategia de Asociatividad Empresarial es importante para la competitividad exportadora ya que se tienen en cuenta a la persona y al empresario, aprovechando los volúmenes de producto para atender los mercados con los cuales se tienen convenios firmados.

Ubilla e Irigoye (2007), Mariño (2012), y Szmulewicz y Gutiérrez (2012) hacen estas publicaciones a partir de investigaciones en las cuales la estrategia de asociatividad es fundamental para mejorar los niveles laborales e incrementar el empleo y la productividad en las actividades económicas de las comunidades objeto de estudio. Los tipos de investigación utilizados fueron cualitativo, cuantitativo y transversal no experimental; se utilizaron instrumentos como fichas, encuestas y entrevistas a cada población. Maldovan (2012) en el artículo "Trabajo, asociatividad y acción colectiva el caso de las cooperativas de recuperadores urbanos" hace un análisis de procesos de la consolidación de cooperativas que se encargaban de la clasificación de residuos sólidos. El autor hace referencia a la asociación y conformación de organizaciones con el fin de mejorar las condiciones laborales y el sistema de higiene urbana de Buenos Aires. En esta investigación participativa se contó con 3000 cartoneros o recuperadores urbanos como población; los resultados muestran que la organización de asociaciones es una buena estrategia empresarial que ayuda a mejorar las condiciones laborales pues se genera empleo y se mejora sustancialmente la calidad de vida de sus comunidad.

Morandé (2006) en la investigación "La Integración de la Sociabilidad y Asociatividad en el Trabajo con Familias Marginales" hace énfasis en la importancia de tales características en la fundación de microempresas, ya que se logran beneficios importantes para la localidad. Esta investigación exploratoria de carácter cualitativo utilizó entrevistas semiestructuradas. Como resultado se muestra una integración de la sociabilidad y asociatividad. Se mostraron resultados positivos para la comunidad ya que se generó empleo, 
se aumentó la producción y se incrementaron las utilidades considerablemente. Igualmente, se resalta que el apoyo emocional es importante para aumentar la confianza al conformar las diversas redes empresariales.

Plazas et al. (2008) en su publicación plantea la importancia de crear un estrategia para mejorar la competitividad de los productores a pequeña escala en el Valle del Cauca. El propósito es innovar e intervenir las agrocadenas con el fin de articular mediante la asociatividad, en empresarios de la región, elementos de tecnología, gestión e inteligencia para mejorar la competitividad en el mercado actual. Como población objetivo de esta investigación acción modo dos se tomaron 300 familias campesinas en los municipios de Popayán, Timbo y Piendamó, entre otros. Los resultados enfatizan la asociación de instituciones, gobiernos locales y académicos de diversos programas con el fin de incrementar la productividad y la competitividad de cada región. Como resultado se muestra el mejoramiento de la infraestructura vial logrando un incremento considerable en las utilidades de los productos que se demandan. Maldovan (2012) y Plazas et al. (2008) mencionan la asociatividad como una estrategia empresarial para la búsqueda de acceso al trabajo formal, en la cual tienen que participar las organizaciones formales y el estado para la consecución de objetivos en una comunidad.

En la propuesta de un modelo asociativo planteado por Olave (2005) en el sector de confecciones del departamento del Atlántico se propuso identificar los factores clave de éxito para las pymes. Se hizo un análisis y un diagnóstico de los diversos ámbitos empresariales mediante un plan de mejoramiento en el cual se propuso un modelo de asociación de Gestión Exportadora. El propósito fue implementar dicho modelo en diferentes empresas que desarrollaran la misma actividad y que fueran más competitivas y productivas en el mercado nacional e internacional; como instrumentos para la investigación, se utilizaron entrevistas semiestructuradas con pregunta abierta. Para la validación se utilizaron un enfoque grupal y unas capacitaciones a los empresarios de la zona; como población objeto de estudio se tomaron treinta Pymes del sector de confecciones del departamento del Atlántico. El investigador plantea en los resultados la falta de compromiso por parte de los empresarios del sector de las confecciones, puesto que se generaron pocos resultados a la hora de aplicar el modelo; se deja planteado la importancia del apoyo por parte de las empresas en las investigaciones aplicadas desde la academia. Estas dos investigaciones toman las cadenas productivas como un mecanismo de cooperación entre las pequeñas y medianas empresas en el que cada una tiene autonomía e independencia, pero trabajando por un objetivo común. Los autores plantean que esta es una estrategia que busca solucionar una problemática social y política de las empresas que desarrollan la misma actividad económica.

Se toma la asociatividad como estrategia empresarial, puesto que es importante determinar que gracias a esta teoría se puede unificar ideas de negocios para ser más productivas en el entorno, en el caso de Sogamoso se trabajó la asociatividad a partir de procesos agroindustriales; debido a la relevancia de la actividad económica del sector agropecuario que presenta el municipio de Sogamoso.

\section{DISCUSIÓN FINAL}

Para el desarrollo de este tipo de investigaciones es importante destacar los estudios relacionados con la estrategia empresarial como eje determinante para la consecución de recursos económicos para este tipo de población, la asociatividad como herramienta para la unificación de actividades económicas con el ánimo de complementación y mejoramiento de los procesos y del mismo modo ser competitivos en el mercado. Es por esto que se analizaron estos tipos de estudios con aras a tomar los más impactantes e implementarlos en la comunidad, dando como resultado la creación de pequeñas empresas dedicadas a la trasformación de procesos agroindustriales en el municipio.

Del mismo Rodríguez (2007) en el desarrollo de emprendimiento y asociatividad desde entorno escolar , siendo determinante para este tipo de población; puesto que es fundamental capacitar a la población en etapa escolar con este tipo de propuestas, incentivarlos a creación y ejecución de ideas innovadoras que con el tiempo mejore la calidad de vida de las familias. Destacado investigaciones actuales se encuentra la realizada por Sánchez et al (2019) relacionada con la planificación para empresas solidarias para la población vulnerable, destacando la importancia de este tipo de estudios puesto que se pueden implementar para el crecimiento del nivel de vida de estos individuos, ratificando la importancia del apoyo de actores como son el gobierno, empresa pública, privada y la academia.

\section{CONCLUSIONES}

De acuerdo con el trabajo presentado y a los resultados obtenidos, se pueden plantear las siguientes conclusiones principales:

1.- El desarrollo de estrategias empresariales en comunidades con problemas socio-económicos es de vital importancia, pues estas tienen el potencial para transformar diferentes variables como la educación, los estilos 
de vida y las actividades económicas. Es decir, dichas estrategias permiten mejorar la calidad de vida de la comunidad.

2.- La estrategia empresarial de asociatividad es fundamental para este tipo de población que desarrolla diferentes actividades económicas sin tener ningún conocimiento y apoyo por parte de entidades gubernamentales, lo cual genera problemas económicos, sociales y culturales en esta comunidad. (Sánchez et al. 2019).

3.- Una vez recopilada la información se destacaron las investigaciones que sirven de soporte para el desarrollo de procesos importantes en este estudio gracias a que muestran la importancia del emprendimiento y asociatividad como estrategia empresarial para la población vulnerable

\section{AGRADECIMIENTOS}

Los autores agradecen a la Universidad Pedagógica y Tecnológica de Colombia por el apoyo al desarrollo del proyecto de investigación realizado por el grupo de investigación CERES.

\section{REFERENCIAS}

Arcoiris, c. n. Monografía Político Electoral departamento de Boyacá. (2008)

Arias, M., L. Portilla, J. Castaño. Modelo asociatividad para producción de piña deshidratada. doi.org/10.22517/23447214.331 Scientia et Technica, xvi (45), 73-78 (2010)

Buvinic, M. The vulnerability of households headed by women: policy questions and options for Latin America and the Caribbean. CEPAL. Retrieved from. (1991)

Calderón, G., C. Álvarez y J. Naranjo. Competitive Strategy and Organizational Performance at Colombian Industrial Companies, Innovar, 20(38), 13-26. (2010)

Cervilla, A., Estrategias para el desarrollo empresarial: Asociatividad en el sector plástico venezolano. Revista de Ciencias Sociales. (Ve), 8(2),230-248. (2007)

DANE, Departamento Administrativo Nacional de Estadística, Población en Colombia (2016)

DNP, Departamento Nacional de Planeación, diálogo regional para Bogotá, enero 21 de 2015 la construcción del plan nacional de desarrollo 2014-2018 (2015)

Engle, P.Strategies for reducing inequalities and improving developmental outcomes for young children in low-income and middle-income countries.doi.org/10.1016/S0140-6736(11)60889-1,The Lancet, 378(9799), 1339-1353. (2011)

Foschiatti, H., Vulnerabilidad Global y Pobreza. Universidad del Noroeste Argentina 1-20 (2008)

Gómez, L. Desempleo y Tasa de Desocupación. Banco de la Republica (2007)

Ibáñez , M. у A. Moya ."Vulnerabilidad del víctimas de los conflictos civiles: Evidencia empírica para la población desplazada en Colombia." Elsevier: 647-663 (2010)

Jaramillo, M., Reubicación y Restablecimiento de la Ciudad.Universidad Humanística 67-72 (2006)

Kingsbury, A. y R. Hayter, Business associations and local development: The Okanagan wine industry's response to NAFTA. Doi:org/10.1016/j.geoforum.2005.12.001, Geoforum, 37(4), 596-609 (2006)

Maldovan, J. Work, associativism and collective action: the case of the recovery and classification of solid waste cooperatives." Trabajo y Sociedad 5(9):139-151 (2012)

Manso,C. Diccionario enciclopédico de estrategia empresarial, 1ra Ed . M. E. D. d. Santos (2003)

Mariño, G. La Associativity as an Entrepreneurial Strategy. Global Conference on Business and Finance Proceedings 7 , 1-7 (2012)

Montoya, A. La formación estratégica en Mintzberg y la posibilidad de una aportación para el futuro. Facultad de Ciencias Administrativas y Contables Universidad Nacional de Colombia, 8(2) 23-44 (2009)

Moore, K. Porter or Mintzberg: whose view of strategy is the most relevant today. Forbes. com. (2011)

Morandé, M. The Integration of Sociability and Active Membership in Social Work with Marginal Families, doi.org/10.4067/S0718-22282006000100009, Psykhe (Santiago), 15(1), 107-117 (2006)

Niño, M., A. Barrientos A., Hickey, S., y D. Hulme. Social Protection in Sub-Saharan Africa: doi.org/10.1016/j.worlddev.2011.04.004. Getting the Politics Right. World Development, 40(1), 163-176. (2012)

Olave, J. Propuesta de un modelo asociativo de gestión exportadora a partir del análisis del sector de confecciones del departamento del Atlántico (Colombia). Pensamiento y Gestión, (19), 141-199 (2005)

Osorio, F., J. Gálvez, J. y G. Murillo La estrategia y el emprendedor: diversas perspectivas para el análisis. Cuadernos de Administración (Universidad Del Valle), (43), 65-80. (2010) 
Pachón, L. D. Una Aproximación a la Vulnerabilidad Bogotá Departamento Nacional de Planeación. (2007)

Pizarro, R. La vulnerabilidad social y sus desafíos: una mirada desde América Latina, Naciones Unidas Cepal Santiago de Chile (2001)

Plazas, A., L. Pemberthy, y D, Sánchez. Hacia El Planteamiento De Una Estrategia Integral Para Mejorar La Competitividad De Productores Rurales De Pequeña Escala En El Cauca - Colombia. Revista Internacional Administración y Finanzas 1-22 (2008)

Porto, M. F. de S., y L, Fernandes. Understanding risks in socially vulnerable contexts: The case of waste burning in cement kilns in Brazil. Safety Science, 44(3), 241-257. https://doi.org/10.1016/j.ssci.2005.10.001 (2018)

Rendón, I. Social entrepreneurship: critical factors of success and failure improvement program for the generation income of the Presidency of the Republic of Colombia aimed at people living in navigating the violence in Caldas. Manizales. (2011)

Rodríguez, L. Emprendimiento y Asociatividad como Herramienta de Desarrollo Social para la Educación Media. Cuadernos Latinoamericanos de Administración II: 65-89 (2007)

Rosenberg, A., K. Hartwig, y M. Merson. Government-NGO collaboration and sustainability of orphans and vulnerable children projects in southern Africa. doi.org/10.1016/j.evalprogplan.2007.08.005 . Evaluation and Program Planning, 31(1), 51-60. (2008)

Sánchez-Otero, M., C. Migdalia, M. Garizabal-Donado y H. Hernández. Organizaciones Solidarias Creadas por población vulnerable: un estudio de su planificación y gestión interna. Información tecnológica, 30(2), 95102. https://dx.doi.org/10.4067/S0718-07642019000200095 (2019)

Szmulewicz, P., C. Gutiérrez y K. Winkler. Partnership and Agritourism. Assessment of Associative Skills in Agrotourism Networks in Southern Chile. Studies and Perspectives in Tourism, 21(4), 1013-1034. (2012)

Thurman, T., R. Snider, y otros cuatro autores. Barriers to the community support of orphans and vulnerable youth in Rwanda. doi.org/10.1016/j.socscimed.2007.12.001. Social Science y Medicine, 66(7), 1557-1567. (2008)

Ubilla, G. y D. Irigoye. Asociatividad de trabajadores cartoneros y Desarrollo Humano Universidad Académica de Humanismo Cristiano191. (2007) 
